University of Wollongong

Research Online

Australian Institute for Innovative Materials -

Papers

Australian Institute for Innovative Materials

2007

\title{
Apparent negative mobility of vortex matter due to inhomogeneous pinning
}

$\mathrm{X} \mathrm{B} \mathrm{Xu}$

Nanjing University, China

H Fangohr

University of Southampton, UK

Shichao Ding

University of Wollongong, sding@uow.edu.au

$\mathrm{M} \mathrm{Gu}$

Department of Physics, Nanjing University, China

T.B Tang

Hong Kong Baptist University, China

See next page for additional authors

Follow this and additional works at: https://ro.uow.edu.au/aiimpapers

Part of the Engineering Commons, and the Physical Sciences and Mathematics Commons

Research Online is the open access institutional repository for the University of Wollongong. For further information contact the UOW Library: research-pubs@uow.edu.au 


\title{
Apparent negative mobility of vortex matter due to inhomogeneous pinning
}

\begin{abstract}
We investigate the transport of vortices in superconductors with inhomogeneous pinning under a driving force. The inhomogeneity of pinning is simplified as strong-weak pinning regions. It is demonstrated that the interactions between the vortices captured by strong pinning potentials and the vortices in the weak pinning region cause absolute negative motion (ANM) of vortices: The vortices which are climbing toward the high barriers induced by the strong pinning with the help of driving force move toward the opposite direction of the force and back to their equilibrium positions in the weak pinning region as the force decreases or is withdrawn. Our simulations reveal that the hysteresis of ANM is determined by the competition between the speed of the negative motion which depends on the pinning inhomogeneity in superconductors and the speed of the driving force. Under the conditions of either larger force scanning rate or higher pinning inhomogeneity, a marked ANM and a larger hysteretic speed-force loop could be observed. This indicates that the time window to observe the ANM should be chosen properly. Moreover, the $\mathrm{V}-\mathrm{I}$ characteristics of $\mathrm{Ag}$-sheathed $\mathrm{Bi}-2223$ tapes are measured, and experimental observations are qualitatively in agreement with the simulation.
\end{abstract}

Keywords

Apparent, negative, mobility, vortex, matter, due, inhomogeneous, pinning

Disciplines

Engineering | Physical Sciences and Mathematics

Publication Details

Xu, XB, Fangohr, H, Ding, S, Gu, M, Tang, TB, Han, Z, Shi, D \& Dou, SX (2007), Apparent negative mobility of vortex matter due to inhomogeneous pinning, Physical Review B (Condensed Matter and Materials Physics), 75 (22), pp. 224507-1-224507-5.

\section{Authors}

X B Xu, H Fangohr, Shichao Ding, M Gu, T.B Tang, Z Han, Dongqi Shi, and S X. Dou

This journal article is available at Research Online: https://ro.uow.edu.au/aiimpapers/216 


\title{
Apparent negative mobility of vortex matter due to inhomogeneous pinning
}

\author{
X. B. Xu, ${ }^{1, *}$ H. Fangohr, ${ }^{2}$ S. Y. Ding, ${ }^{1}$ M. Gu, ${ }^{1}$ T. B. Tang, ${ }^{3}$ Z. H. Han, ${ }^{4}$ D. Q. Shi, ${ }^{5}$ and S. X. Dou ${ }^{5}$ \\ ${ }^{1}$ National Laboratory of Solid State Microstructures, Department of Physics, Nanjing University, Nanjing 210093, \\ People's Republic of China \\ ${ }^{2}$ School of Engineering Sciences, University of Southampton, Southampton SO17 1BJ, United Kingdom \\ ${ }^{3}$ Department of Physics, Hong Kong Baptist University, Kowloon, Hong Kong SAR, People's Republic of China \\ ${ }^{4}$ Applied Superconductivity Research Center, Tsinghua University, Beijing 100084, People's Republic of China \\ ${ }^{5}$ Institute for Superconducting and Electronic Materials, University of Wollongong, New South Wales 2522, Australia
}

(Received 17 January 2007; revised manuscript received 9 May 2007; published 13 June 2007)

\begin{abstract}
We investigate the transport of vortices in superconductors with inhomogeneous pinning under a driving force. The inhomogeneity of pinning is simplified as strong-weak pinning regions. It is demonstrated that the interactions between the vortices captured by strong pinning potentials and the vortices in the weak pinning region cause absolute negative motion (ANM) of vortices: The vortices which are climbing toward the high barriers induced by the strong pinning with the help of driving force move toward the opposite direction of the force and back to their equilibrium positions in the weak pinning region as the force decreases or is withdrawn. Our simulations reveal that the hysteresis of ANM is determined by the competition between the speed of the negative motion which depends on the pinning inhomogeneity in superconductors and the speed of the driving force. Under the conditions of either larger force scanning rate or higher pinning inhomogeneity, a marked ANM and a larger hysteretic speed-force loop could be observed. This indicates that the time window to observe the ANM should be chosen properly. Moreover, the $V-I$ characteristics of Ag-sheathed Bi-2223 tapes are measured, and experimental observations are qualitatively in agreement with the simulation.
\end{abstract}

DOI: 10.1103/PhysRevB.75.224507

PACS number(s): 74.25.Fy, 74.25.Qt

When applying a force to a static body, it will usually move along the direction of the force. An anomalous behavior termed as absolute negative motion (ANM) is that the motion is toward the opposite direction of the force. ANM is expected to occur for the particles confined in an asymmetric potential (i.e., so-called ratchet potential) and subject to an unbiased fluctuating drive. Thus, ANM has been mostly studied under an alternating external force, such as ac drive in superconducting systems. ${ }^{1-7}$ Practically, ANM may also take place for dc drive. For example, several groups have observed the ANM which manifests itself by negative voltage or resistance in transport measurements of voltage-current or resistance-temperature characteristics in superconductors, such as bulk $\mathrm{YBaCuO}$ and $\mathrm{BiPbSrCaCuO},{ }^{8}$ sintered YBaCuO, ${ }^{9}$ granular Sn (Ref. 10) or Al films, ${ }^{11}$ and larger arrays of vanadium wires covered with Au or Fe. ${ }^{12}$ Different mechanisms or models, including thermoelectric effect, ${ }^{8}$ weak-link effect (causing redistribution of vortices), ${ }^{9}$ and the electrical contacts' arrangement in combination with the superconducting proximity effect, ${ }^{12}$ have been proposed to explain this fascinating phenomenon in vortex systems. ${ }^{8,9,12}$ In spite of considerable experimental effort, the ANM connected with vortex motion is still far from being completely understood. Specifically, the numerical investigation has been sparse so far.

The above-mentioned experimental observations have given two important clues to explain this anomalous phenomenon. First, for the polycrystaline samples, such as AgBi2223 tapes, magneto-optical imaging of vortex patterns in the $\mathrm{Bi} 2223$ composites reveals that the strong vortex pinning occurs at the Ag-Bi2223 interface where the effective texturing takes place, the weak pinning (smaller critical current density $j_{c}$ ) appearing to be near the core of the filaments. ${ }^{13}$ As a matter of fact, inhomogeneous vortex pinning models have been developed and successfully explained experiments. ${ }^{14}$ Furthermore, even for a sample with homogeneous pinning (i.e., with constant $j_{c}$ ) strong surface barriers may exist, ${ }^{15}$ which is confirmed by experiments and simulation. ${ }^{16,17}$ The surface barriers imply higher surface $j_{c}$. In other words, vortex pinning strength is distinct in the edges from the center in these samples. Second, at higher temperature where ANM has frequently been observed, weak pinning potentials can easily be smeared out by thermal fluctuations. So, the localized strong pinning will be more effective to capture vortices than weak pointlike pinning. ${ }^{16}$ The resulting local current density $j$ might exceed a critical value $j_{c}$, and then the vortices rearrange such that $j \leqslant j_{c}$ everywhere in the superconductors. ${ }^{15}$ Therefore, the inhomogeneous vortex pinning models (including strong pinning inside a superconductor ${ }^{14}$ and surface barriers ${ }^{16,17}$ ) may be helpful in understanding the underlying physics in the ANM.

In fact, the vortex motion itself is of great interest as the localized strong pinning centers (such as twin boundaries, artificial columnar defects, and so on) occur in superconductors. It is evident that as one part of vortices is trapped in the regime with strong pinning, the motion of the others will be intensely influenced by these trapped vortices due to intervortex interactions. ${ }^{2,5}$ Once these interactions are more important than either external drive or thermal fluctuations, the resulting macroscopic transport behaviors can be quite different from the behaviors in superconductors with homogeneous pinning such as uncorrelated pointlike pins.

In this work, in order to obtain an understanding of the ANM of vortex systems for dc drive at microscopic level, we numerically study the ANM via molecular dynamics simulation based on strong-weak pinning model. Besides, to check our numerical results, the $V-I$ characteristics of Ag-sheathed Bi2223 tapes are measured. 


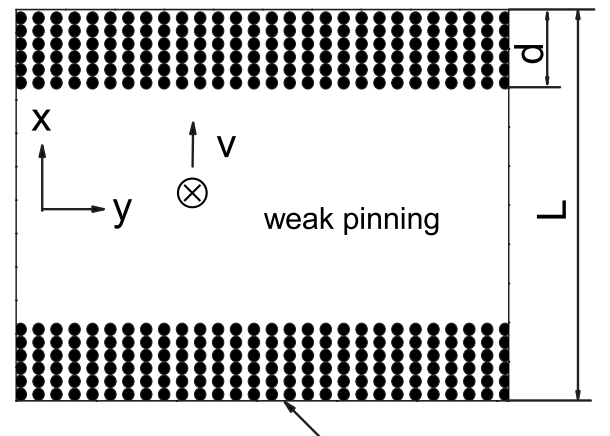

strong pinning

FIG. 1. Sketch of the $x y$ section of the simulation box at $z=0$. The black spots and empty squares denote the strong and weak pinning centers, respectively. The Lorentz force is in the $x$ direction, and magnetic field is in the $z$ direction.

The schematic plot of our inhomogeneous vortex pinning model is shown in Fig. 1, which is characterized by different regions with stronger $f_{p v 0}$ and weaker $f_{w}$ pinning forces. The difference $f_{p v 0}-f_{w}$ shows the pinning inhomogeneity. We let $f_{w}=0$ in the next context, and study the evolution of the ANM at variable pinning inhomogeneity by adjusting $f_{p v 0}$. We have also studied the effects of finite random pinning in the weak pinning region. The corresponding simulation results (not shown here) suggest that the weak pinning does not affect qualitatively our main results on the ANM. The driving force is in the $x$ direction, while the applied current and magnetic field are in $y$ and $z$ directions (perpendicular to the paper face), respectively, in the infinite plane. The vortices will move along the $x$ direction as the driving force is large enough.

The overdamped Langevin equation of motion for a vortex in position $\mathbf{r}_{i}$ is ${ }^{18}$

$$
\mathbf{F}_{i}=\sum_{j \neq i}^{N_{v}} \mathbf{F}^{v v}\left(\mathbf{r}_{i}-\mathbf{r}_{j}\right)+\sum_{k}^{N_{p}} \mathbf{F}^{v p}\left(\mathbf{r}_{i}-\mathbf{r}_{k}^{p}\right)+\mathbf{F}^{\mathrm{L}}+\mathbf{F}_{i}^{\mathrm{T}}=\eta \frac{d \mathbf{r}_{i}}{d t},
$$

where $\mathbf{F}_{i}$ is the total force acting on vortex $i, \mathbf{F}^{v v}$ and $\mathbf{F}^{v p}$ are the forces due to vortex-vortex and vortex-pin interactions, respectively, $\mathbf{F}^{\mathrm{L}}$ is the driving force due to the current $\mathbf{J}$ $\left(\mathbf{F}^{\mathrm{L}} \propto \mathbf{J} \times \hat{\mathbf{z}}\right)$ and $\mathbf{F}^{\mathrm{T}}$ is the thermal stochastic force, $\eta$ is the Bardeen-Stephen friction coefficient $\left(\eta \propto \phi_{0} B_{c 2} / \rho_{n}\right), N_{v}$ is the number of vortices, $N_{p}$ is the number of pinning centers, and $\mathbf{r}_{k}^{p}$ is the position of the $k$ th pinning center. We choose $\mathbf{F}^{v v}\left(\mathbf{r}_{i}-\mathbf{r}_{j}\right)=\left(\phi_{0}^{2} s\right)\left(2 \pi \mu_{0} \lambda^{2}\right)^{-1}\left(\mathbf{r}_{i}-\mathbf{r}_{j}\right)\left(\left|\mathbf{r}_{i}-\mathbf{r}_{j}\right|\right)^{-2}$, where $\phi_{0}$ is the flux quantum, $s$ the length of the vortex, and $\mu_{0}$ the vacuum permeability. We employ periodic boundary conditions and cut off the logarithmic vortex-vortex repulsion potential smoothly. ${ }^{19}$ Pinning centers exert an attractive force on the vortices: $\mathbf{F}^{v p}\left(\mathbf{r}_{i}-\mathbf{r}_{k}^{p}\right)=-f_{p v 0} r_{i k} \exp \left[-\left(r_{i k} / r_{p}\right)^{2}\right] \hat{\mathbf{r}}_{i k} / r_{p}$, where $f_{p v 0}$ tunes the strength of this force at zero temperature, and $r_{p}$ which was assumed to equal to $0.2 \lambda$ determines its range. The thermal force has properties $\left\langle F_{i}^{\mathrm{T}}\right\rangle=0$ and $\left\langle F_{i}^{\mathrm{T}}(t) F_{j}^{\mathrm{T}}\left(t^{\prime}\right)\right\rangle=2 \eta k_{B} T \delta_{i j} \delta\left(t-t^{\prime}\right)$ at a given temperature $T$, where $k_{B}$ is the Boltzmann constant. To reflect the competing roles of $\mathbf{F}^{v v}, \mathbf{F}^{v p}$, and $\mathbf{F}^{\mathrm{T}}$ as a function of temperature, we use $\lambda(T) / \lambda(0)=\xi(T) / \xi(0)=\left(1-T / T_{c}\right)^{-1 / 2} \cdot{ }^{20}$ The average veloci- ties of vortices is $\left\langle V_{x}\right\rangle=\frac{1}{N} \sum_{i}^{N_{v}} v_{i x}$ which is proportional to the mobility of the vortices and the resulting voltage. We normalize lengths by $\lambda_{0}=\lambda(0)$, the forces by $f_{0}=\left(\phi_{0}^{2} s\right)$ $\times\left(2 \pi \mu_{0} \lambda_{0}^{3}\right)^{-1}$, and time by $t_{0}=\lambda_{0} \eta(0) / f_{0}$. All quantities shown in the following figures are expressed in simulation units. The same initial vortex states are obtained by annealing and then subjected to a Lorentz driving force along the $x$ axis. $N_{v}=900$ is chosen in this simulation. For larger systems similar results are observed. Besides, we employ $B$ $=0.03 B_{c 20}, T=0.8 T_{c}, \lambda_{0}=690 \AA, s=0.25 \times 10^{-2} \mathrm{~m}$, and $\eta$ $=1 \times 10^{-11} \mathrm{~kg} / \mathrm{s}$.

Shown in Fig. 2(a) is a typical and clockwise hysteretic $V_{x}-F^{\mathrm{L}}$ loop in one circle and the corresponding vortex moving trajectories. The inset is the $V_{x}-t$ (velocity-time) curve. Let us now concentrate on the descending branch of the loop. It is clear that there is an apparent negative velocity region of vortex motion as driving force is lower than the $F_{B}$ $\approx 0.65 f_{0}$ which is the Lorentz force at point $B$. We define $F_{B}$ to be the value of the driving force at which the observed average vortex velocity in $x$ direction is zero, i.e., $\left\langle v_{x}\left(F_{B}\right)\right\rangle$ $=0$. For large driving forces $F^{\mathrm{L}}>F_{B}$, the average velocity of vortices is positive: the direction of moving vortices is the same as that of the driving force, as seen in Fig. 2(b). For $F^{\mathrm{L}}=F_{B}$, although the driving force is still positive, the vortex average velocity is zero. On the descending branch for $F^{\mathrm{L}}$ $<F_{B}$, the repelling forces from the captured vortices overcome the positive driving force. Then, the moving vortices will go back to their equilibrium positions in the weak pinning region, shown by the arrows in Fig. 2(c). The resulting motion of vortices is in the opposite direction of the driving force, which is therefore termed as the apparent negative motion of vortices for dc drive. Besides, as the descending driving force reaches zero, the negative velocity reaches its maximum [see the inset of Fig. 2(a)]. Then, the speed of vortices gradually decreases to zero during a time interval of about $20 t_{0}$, which is the waiting time for the vortices to move back to their equilibrium positions.

Figure 3 demonstrates the effect of current or driving force scanning rate (CSR) on the ANM (also the hysteresis of $V_{x}-F^{\mathrm{L}}$ characteristics). The more pronounced the ANM effect, the higher the current scanning rate. This simulation result indicates that to make the ANM and the hysteretic $V-I$ curves experimentally accessible, the experimental speed should be fast enough compared with the reverse diffusion velocity of vortices. ${ }^{22}$ That is, the experimental time window to observe the ANM has to be chosen properly.

Figure 4 shows the effect of $d / L$ on the ANM of vortex systems. For larger $d / L$, on the one hand, more vortices will be captured by the strong pinning. This leads to larger repelling forces exerting on the vortices moving reversely in the weak region. On the other hand, the larger $d / L$ also means that there are fewer vortices in the weak pinning region at a given magnetic field, and they could only make a shorter reversal journey before reaching their equilibrium positions (because $L$ is unchanged at a fixed field). Apparently, the latter effects on the ANM are more important. Therefore, the ANM and the size of $V_{x}-F^{\mathrm{L}}$ hysteretic loop decrease with increasing $d / L$.

The effect of pinning inhomogeneity on the ANM is 

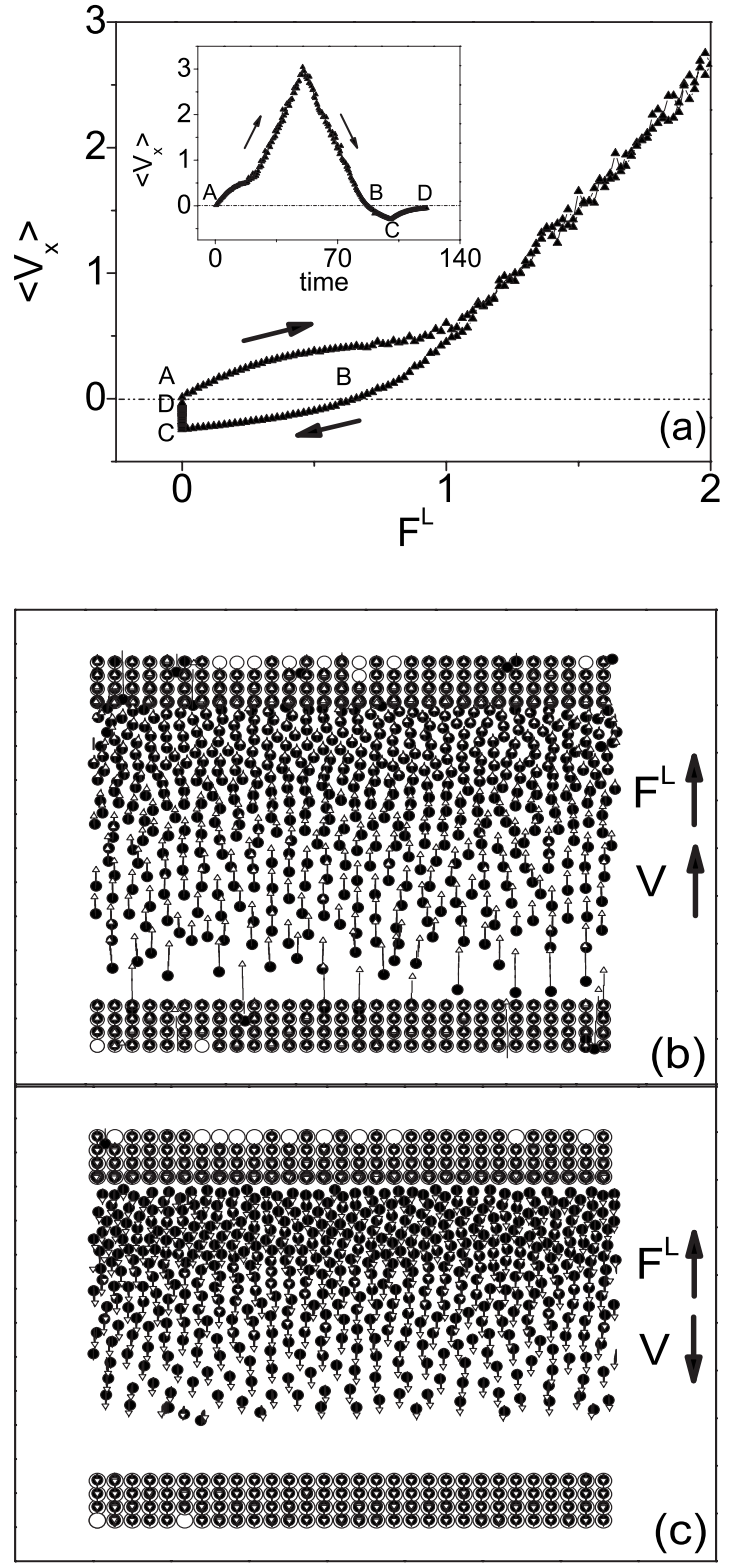

FIG. 2. (a) A numerical clockwise $V_{x}-F^{\mathrm{L}}$ loop. The arrows indicate the evolution of the driving force. The inset displays the time evolution of the average velocity of vortices. It is noted that negative velocity occurs when the driving force is decreasing and small. (b) Vortex trajectories [from beginning $(\bullet)$ to end $(\triangle)$ ] for driving forces from $F^{\mathrm{L}}=F_{B}+0.26 f_{0}$ to $F^{\mathrm{L}}=F_{B}$ in the descending branch. The vortices are moving in the same direction as the driving force. The open circles are pinning centers. (c) Vortex trajectories [from beginning $(\circlearrowleft)$ to end $(\nabla)$ ] for driving forces from $F^{\mathrm{L}}=F_{B}$ to $F^{\mathrm{L}}$ $=F_{B}-0.26 f_{0}$ in the descending branch. In this case, due to the fact that the repelling forces from the captured vortices by strong pinning are larger than $F^{L}$, the vortices in weak pinning region are drifting in the opposite direction of the driving force and return to their equilibrium positions. We have used $f_{p v 0}=30 f_{0}, d F^{\mathrm{L}} / d t$ $=0.01 f_{0} / t_{0}$, and $d / L=4 / 30$.

shown in Fig. 5. As mentioned above, larger $f_{p v 0}$ corresponds to larger pinning inhomogeneity. For increasing $f_{p v 0}<30 f_{0}$, more vortices are captured in the strong pinning region. This results in larger repelling forces exerted on the vortices in the

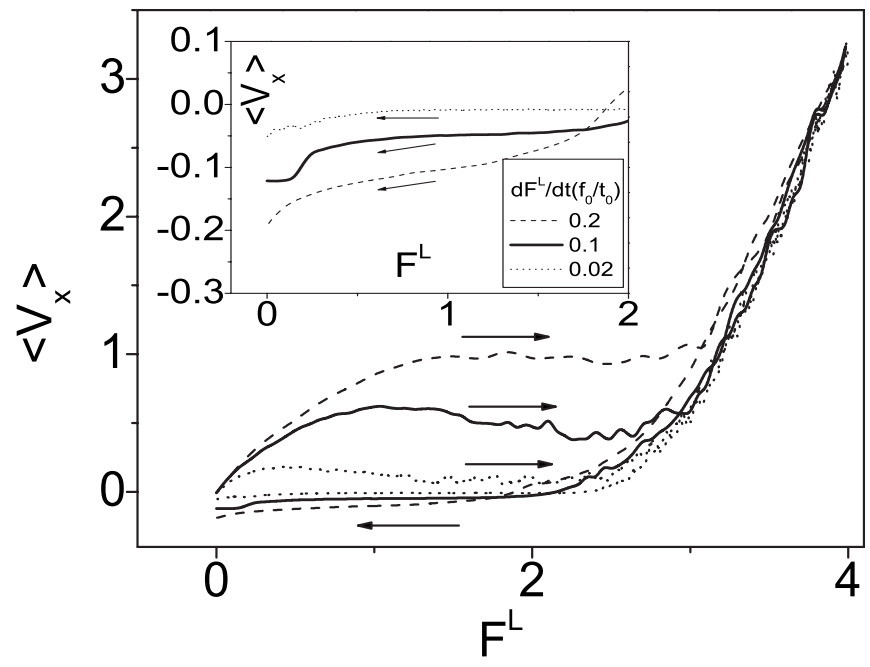

FIG. 3. Effect of driving force scanning rate on the apparent negative motion (with $f_{p v 0}=60 f_{0}, d / L=6 / 30$ ). The inset shows the magnification of the descending branch of $V-F^{\mathrm{L}}$ loop for low $F^{\mathrm{L}}$. ANM and hysteresis loop become invisible with the decreasing scanning rate. It is clear that the negative motion and the hysteresis loop are more apparent at fast scanning rate than at slow rate.

weak region, which leads to more pronounced ANM. Figure 5 shows that the ANM is most pronounced for $f_{p v 0}=30 f_{0}$. If $f_{p v 0}$ is further increased to $30 f_{0}$, the ANM decreases due to fewer vortices available that could move reversibly in the weak pinning region. This simulation result implies that the ANM and the hysteresis loop could be more pronounced for a sample with larger portion of weak links. ${ }^{12}$ It could also be used to explain the fact that ANM has not been reported by now for single crystals with weak and homogeneous vortex pinning.

We compare the simulations with experiments. Electrical transport measurement was conducted using the four-probe method on $\mathrm{Ag}$-sheathed $\mathrm{Bi}_{2-x} \mathrm{~Pb}_{x} \mathrm{Sr}_{2} \mathrm{Ca}_{2} \mathrm{Cu}_{3} \mathrm{O}_{y}$ tapes im-

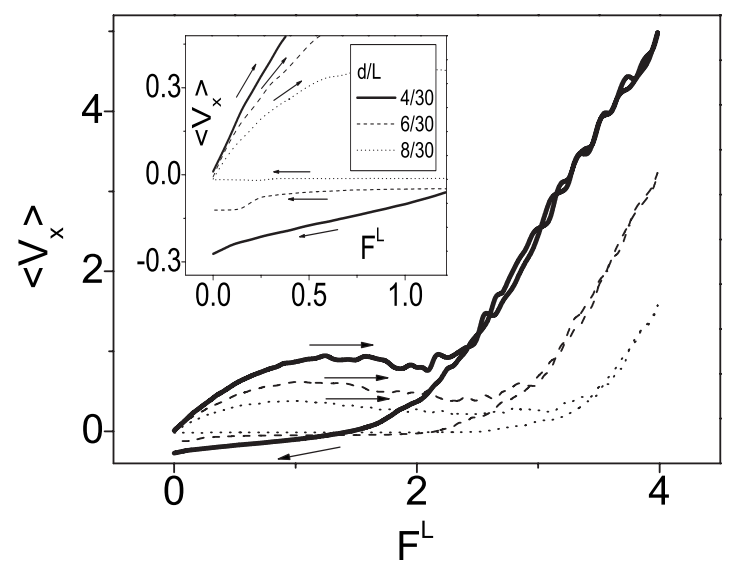

FIG. 4. Effect of $d / L$ on the apparent negative motion. The larger $d / L$ means that there are fewer vortices in the weak pinning region and they will only make a shorter reversal journey before reaching their equilibrium positions (thus having a shorter relaxation time). Therefore, the larger $d / L$ is, the smaller the ANM (also the hysteretic $V-F^{\mathrm{L}}$ loop) is. $f_{p v 0}=60 f_{0}$ and $d F^{\mathrm{L}} / d t=0.1 f_{0} / t_{0}$. The inset shows the magnification of the $V-F^{\mathrm{L}}$ curve for low $F^{\mathrm{L}}$. 


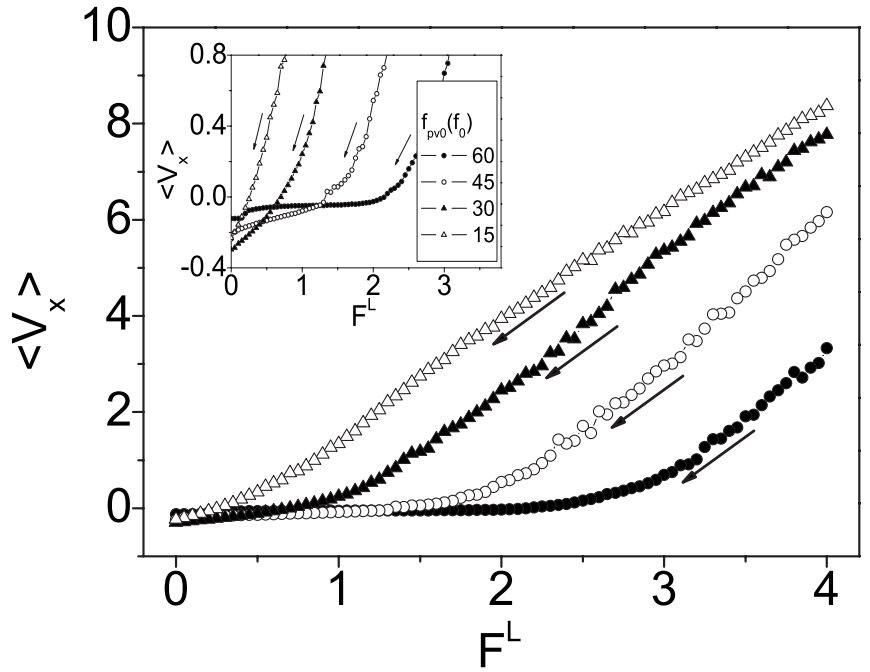

FIG. 5. Effect of the inhomogeneity of pinning strength $f_{p v 0}$ on ANM and size of the hysteresis loop. $d / L=6 / 30$ and $d F^{\mathrm{L}} / d t$ $=0.1 f_{0} / t_{0}$. The inset shows the magnification of the $V-F^{\mathrm{L}}$ curve for low $F^{\mathrm{L}}$. It is noted that ANM and the loop become too small to observe for very small $f_{p v 0}$.

mersed in liquid nitrogen in magnetic fields. These tapes represent a system with the type of inhomogeneous pinning studied here. All the samples have approximately the same size, i.e., $L_{a} \times L_{b} \times L_{c}=3 \mathrm{~cm} \times 0.5 \mathrm{~cm} \times 0.25 \mathrm{~cm}$, where the textured $c$ axis is perpendicular to the wider surfaces of the samples. The distance between two voltage leads is $L_{v}$ $=1 \mathrm{~cm}$ and the distance between two current leads is $3 \mathrm{~cm}$, each of which is $1 \mathrm{~cm}$ away from the voltage leads. The details of the sample preparation and measurement can be found in Ref. 23.

Illustrated in Fig. 6 are the effects of CSR on ANM for one of the samples. It is seen that the largest ANM is observed at the highest CSR $\left(4.61 I_{c} / \mathrm{min}\right)$, which is qualitatively in agreement with the simulation shown in Fig. 3. The experimentally measured ANM shown in Fig. 6 is changing as a function of the CSR, and in agreement with our simulation results. Consequently, the previously proposed mechanisms leading to ANM including thermoelectric effect ${ }^{8}$ and the electrical contacts' arrangement in combination with the superconducting proximity effect, ${ }^{12}$ are unlikely to explain the occurrence of the ANM shown in Fig. 6. In addition, Fig. 6 displays that the critical current density decreases with the increasing CSR, which is consistent with the experimental and numerical observations. ${ }^{14,23}$

The evolution of the ANM with applied magnetic field is shown in Fig. 7. The vortex-vortex interactions are increasing with the increasing magnetic field, which leads to a reduction in the number of trapped vortices by the localized strong pinning. The repelling forces exerted on the reversibly drifting vortices are accordingly decreasing. Therefore, the ANM and also the size of hysteretic $V$ - $I$ loops decrease with increasing field.

For the quantitative comparison of the experimental data with the simulation results, we convert the experimental data into simulation units. The conversion factor from dimensionless simulation units to vortex velocity in $\mathrm{m} / \mathrm{s}$ is $c_{\mathrm{vel}}=\lambda_{0} / t_{0}$

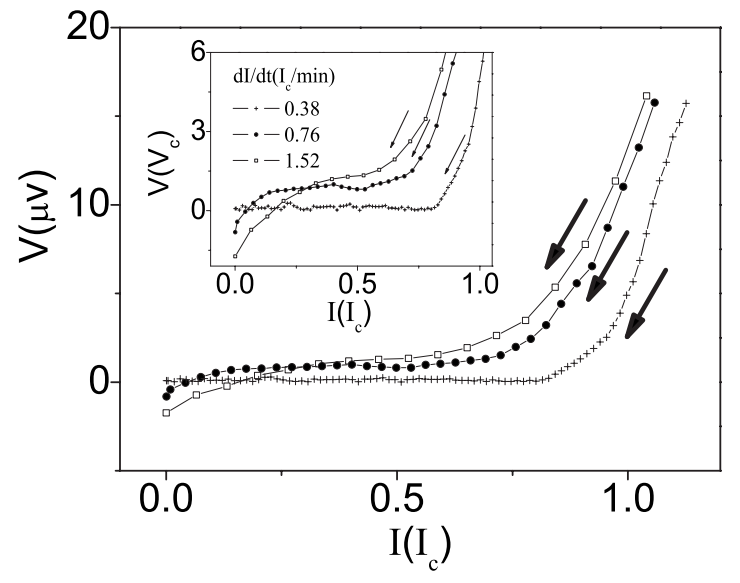

FIG. 6. Experimental $V-I$ curves upon descending current (the ascending branches are not shown here for clarity) for different $d I / d t$ and at temperature $T=77 \mathrm{~K}$ and reduced field $H / H_{c 2}=8.33$ $\times 10^{-3}$. It is seen that negative voltage occurs for higher CSR, which is in good agreement with the numerical results shown in Fig. 3. The inset shows the enlarged view of the $V-I$ curves. $I_{c}$ $=13 \mathrm{~A}$ which is the typical critical current at lower CSR for our samples, and the voltage criterion is $V_{c}=1 \mu \mathrm{V} . H_{c 2}(T=77 \mathrm{~K})$ $=0.6 \mathrm{~T}$ for field parallel to the $c$ axis of $\mathrm{Ag} / \mathrm{Bi}-2223$ polycrystal tape (Ref. 21).

$\approx 3 \times 10^{6} \mathrm{~m} / \mathrm{s}$, and the conversion factor for the driving force is $c_{\text {Lor }}=f_{0} \approx 4 \times 10^{-6} \mathrm{~kg} \mathrm{~m} / \mathrm{s}^{2}$.

Taking a typical experimental pair of values, such as the curve corresponding to $d I / d t=0.76 I_{c} / \mathrm{min}$ in Fig. 6 , we find that a current of $I \approx I_{c}(=13 \mathrm{~A})$ results in a voltage of $V$ $\approx 17 \mu \mathrm{V}$. Expressing these numbers in simulation units, we have a (dimensionless) driving force $F^{\exp }=I \Phi_{0} / L_{b} / f_{0} \approx 2$ $\times 10^{-7}$ and a dimensionless vortex velocity of $v^{\exp }$ $=V /\left(B L_{v}\right) / c_{\mathrm{vel}} \approx 4 \times 10^{-7}$. These numbers need to be interpreted as follows: The driving force used in the simulation is larger than that used in the experiment by a factor of the order of $10^{7}$. The reason for this overexaggerated driving force is that the time window that can be captured by a computer simulation is of the order of $1 \times 10^{6}$ time steps and that the time step size must not exceed a certain value to

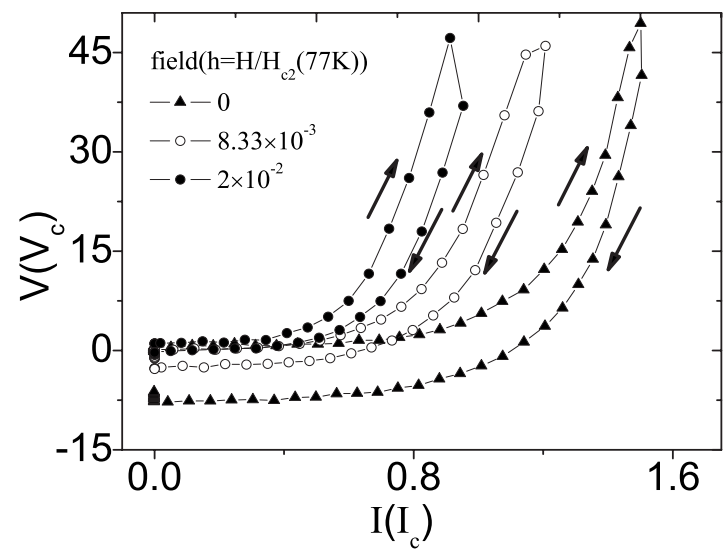

FIG. 7. Experimental $V-I$ curves at different reduced fields. The ANM and the size of hysteretic $V-I$ loops decrease with field. $T$ $=77 \mathrm{~K}$ and $d I / d t=9.22\left(I_{c} / \mathrm{min}\right)$. 
keep the time integration stable. Consequently, any physics that one wishes to observe needs to be accelerated to happen within that time window. We would therefore expect-if the simulation qualitatively represents the dominating physicsthat the resulting vortex velocities in the simulations are also significantly larger than those in the experiment. Indeed, the difference in the observed velocities between experiment and simulation is of the order of $10^{7}$, in agreement with the same order of magnitude difference in the driving force.

The acceleration of the dynamics (by using a much too large driving force) is necessary to study the system and common practice in vortex state simulations due to the reasons outlined above (see, for example, Ref. 18).

In summary, we have proposed a model and performed simulation to study the apparent negative motion of vortices under a dc drive in superconductors with inhomogeneous vortex pinning. It is shown that the vortices which are climb- ing toward the high barriers induced by the strong pinning move toward the opposite direction of the driving force and back to their equilibrium positions in the weak pinning region as the force is decreases or is withdrawn. The simulation suggests that the time window to observe the ANM should be chosen properly. For larger CSR or higher pinning inhomogeneity, the marked ANM and a larger hysteretic $V_{x}-F^{\mathrm{L}}$ loop could be observed. Moreover, measurements of $V$-I characteristics were conducted for Ag-sheathed Bi2223 samples and the experimental results are qualitatively in agreement with the simulation.

This work was supported by grants from the MOST 973 Program of China (No. 2006CB705600), the NSF of China (No. 10674060), and the Jiangsu Province Foundation of Natural Science (No. BK2006109).
*Corresponding author; xxb@nju.edu.cn

${ }^{1}$ J. F. Wambaugh, C. Reichhardt, C. J. Olson, F. Marchesoni, and Franco Nori, Phys. Rev. Lett. 83, 5106 (1999).

${ }^{2}$ J. E. Villegas, S. Savel'ev, F. Nori, E. M. Gonzalez, J. V. Anguita, R. Garia, and J. L. Vicent, Science 302, 1188 (2003).

${ }^{3}$ S. Savel'ev, F. Marchesoni, and Franco Nori, Phys. Rev. Lett. 92, 160602 (2004).

${ }^{4}$ B. Y. Zhu, F. Marchesoni, and Franco Nori, Phys. Rev. Lett. 92, 180602 (2004).

${ }^{5}$ C. C. de Souza Silva, J. Van de Vondel, M. Morelle, and V. V. Moshchalkov, Nature (London) 440, 651 (2006).

${ }^{6}$ C. C. de Souza Silva, J. Van de Vondel, B. Y. Zhu, M. Morelle, and V. V. Moshchalkov, Phys. Rev. B 73, 014507 (2006).

${ }^{7}$ M. Chinappi, E. De Angelis, S. Melchionna, C. M. Casciola, S. Succi, and R. Piva, Phys. Rev. Lett. 97, 144509 (2006).

${ }^{8}$ M. Polák, P. Kottman, M. Majoroš, M. Kedrová, and V. Plecháček, Supercond. Sci. Technol. 3, 67 (1990).

${ }^{9}$ L. F. Goodrich, J. Moreland, and A. Roshko, IEEE Trans. Appl. Supercond. 27, 1194 (1991).

${ }^{10}$ A. V. Herzog, P. Xiong, and R. C. Dynes, Phys. Rev. B 58, 14199 (1998).

${ }^{11}$ C. Strunk, V. Bruyndoncx, C. Van Haesendonck, V. V. Moshchalkov, Y. Bruynseraede, C. J. Chien, B. Burk, and V. Chandrasekhar, Phys. Rev. B 57, 10854 (1998).

${ }^{12}$ J. Jorritsma and J. A. Mydosh, Phys. Rev. B 62, 9703 (2000).

${ }^{13}$ U. Welp, D. O. Gunter, G. W. Crabtree, J. S. Luo, V. A. Maroni, W. L. Carter, V. K. Vlasko-Vlasov, and V. I. Nikitenko, Appl. Phys. Lett. 66, 1270 (1995).

${ }^{14}$ Y. Liu, H. Luo, X. Leng, Z. H. Wang, L. Qiu, S. Y. Ding, and L. Z. Lin, Phys. Rev. B 66, 144510 (2002).

${ }^{15}$ E. H. Brandt and M. Indenbom, Phys. Rev. B 48, 12893 (1993).

${ }^{16}$ Y. Paltiel, E. Zeldov, Y. N. Myasoedov, H. Shtrikman, S. Bhattacharya, M. J. Higgins, Z. L. Xiao, E. Y. Andrei, P. L. Gammel, and D. J. Bishop, Nature (London) 403, 398 (2000); M. Konc- zykowski, L. I. Burlachkov, Y. Yeshurun, and F. Holtzberg, Phys. Rev. B 43, 13707(R) (1991); Y. Paltiel, E. Zeldov, Y. Myasoedov, M. L. Rappaport, G. Jung, S. Bhattacharya, M. J. Higgins, Z. L. Xiao, E. Y. Andrei, P. L. Gammel, and D. J. Bishop, Phys. Rev. Lett. 85, 3712 (2000); Z. L. Xiao, E. Y. Andrei, Y. Paltiel, E. Zeldov, P. Shuk, and M. Greenblatt, Phys. Rev. B 65, 094511 (2002).

${ }^{17}$ X. Leng, S. Y. Ding, Y. Liu, Z. H. Wang, H. K. Liu, and S. X. Dou, Phys. Rev. B 68, 214511 (2003).

${ }^{18}$ See, e.g., C. J. Olson, C. Reichhardt, R. T. Scalettar, G. T. Zimányi, and Niels Grønbech-Jensen, Phys. Rev. B 67, 184523 (2003); C. J. Olson, C. Reichhardt, and Franco Nori, Phys. Rev. Lett. 81, 3757 (1998); Seungoh Ryu, M. Hellerqvist, S. Doniach, A. Kapitulnik, and D. Stroud, ibid. 77, 5114 (1996); C. Reichhardt, G. T. Zimányi, and Niels Grønbech-Jensen, Phys. Rev. B 64, 014501 (2001); H. Fangohr, S. J. Cox, and P. A. J. de Groot, ibid. 64, 64505 (2001); A. B. Kolton, D. Domínguez, and Niels Grønbech-Jensen, Phys. Rev. Lett. 83, 3061 (1999); K. Moon, R. T. Scalettar, and G. T. Zimányi, ibid. 77, 2778 (1996).

${ }^{19}$ H. Fangohr, A. Price, S. Cox, P. A. J. de Groot, G. J. Daniell, and K. S. Thomas, J. Comput. Phys. 162, 372 (2000).

${ }^{20}$ G. Blatter, M. V. Feigel'man, V. B. Geshkenbein, A. I. Larkin, and V. M. Vinokur, Rev. Mod. Phys. 66, 1125 (1994); E. H. Brandt, Rep. Prog. Phys. 58, 1465 (1995).

${ }^{21}$ W. M. Chen, F. Wang, S. S. Jiang, H. K. Liu, and S. X. Dou, J. Supercond. 14, 465 (2001); F. Wang, F. Lin, X. J. Jia, F. X. Han, and W. M. Chen, Chin. J. Low Temp. Phys. 22, 36 (2000).

${ }^{22}$ X. B. Xu, Y. Liu, H. Fangohr, L. Zhang, S. Y. Ding, Z. H. Wang, S. L. Liu, G. J. Wu, and H. M. Shao, Phys. Rev. B 73, 214521 (2006); X. B. Xu, L. Zhang, S. Y. Ding, X. Leng, Z. H. Wang, and X. L. Wang, Supercond. Sci. Technol. 18, 758 (2005).

${ }^{23}$ S. Y. Ding, C. Ren, X. X. Yao, Y. Sun, and H. Zhang, Cryogenics 38, 809 (1998). 